\title{
Infected splenic dendritic cells are sufficient for prion transmission to the CNS in mouse scrapie
}

\author{
Pierre Aucouturier, ${ }^{1,2}$ Frédéric Geissmann, ${ }^{1}$ Diane Damotte, ${ }^{1}$ Gabriela P. Saborio, ${ }^{3}$ \\ Harry C. Meeker, ${ }^{4}$ Regina Kascsak, ${ }^{4}$ Richard Kascsak, ${ }^{4}$ Richard I. Carp, ${ }^{4}$ \\ and Thomas Wisniewski²
}

\author{
${ }^{1}$ Institut National de la Santé et de la Recherche Médicale (INSERM) U25 and \\ Centre National de la Recherche Scientifique U8603, Hôpital Necker, Paris, France \\ ${ }^{2}$ New York University School of Medicine, Department of Neurology, New York, New York, USA \\ ${ }^{3}$ Serono Pharmaceutical Research Institute, Geneva, Switzerland \\ ${ }^{4}$ Institute for Basic Research in Developmental Disabilities, Staten Island, New York, USA
}

Address correspondence to: Pierre Aucouturier, INSERM U25, Hôpital Necker, 161 rue de Sèvres, F-75015 Paris, France. Phone: 33-1-44-49-53-75; Fax: 33-1-44-49-53-74; E-mail: aucouturier@necker.fr.

Received for publication April 30, 2001, and accepted in revised form July 3, 2001.

Transmissible spongiform encephalopathies display long incubation periods at the beginning of which the titer of infectious agents (prions) increases in peripheral lymphoid organs. This "replication" leads to a progressive invasion of the CNS. Follicular dendritic cells appear to support prion replication in lymphoid follicles. However, the subsequent steps of neuroinvasion remain obscure. $\mathrm{CD} 11 \mathrm{c}^{+}$dendritic cells, an unrelated cell type, are candidate vectors for prion propagation. We found a high infectivity titer in splenic dendritic cells from prion-infected mice, suggesting that dendritic cells carry infection. To test this hypothesis, we injected $R A G-1 \% / 0$ mice intravenously with live spleen cell subsets from scrapie-infected donors. Injection of infected dendritic cells induced scrapie without accumulation of prions in the spleen. These results suggest that $\mathrm{CD} 11 \mathrm{c}^{+}$dendritic cells can propagate prions from the periphery to the CNS in the absence of any additional lymphoid element.

J. Clin. Invest. 108:703-708 (2001). DOI:10.1172/JCI200113155.

\section{Introduction}

Prions, which are responsible for transmissible spongiform encephalopathies in humans and animals, are unconventional infectious agents essentially composed of a modified host protein termed $\operatorname{PrP}(1)$. One of the unusual features of prion infections is a long incubation period that precedes invasion of the CNS. The pathophysiological events occurring at this preclinical step influence subsequent neurological evolution and are, therefore, potential therapeutic targets. The natural history of the disease has been mainly studied in mouse experimental scrapie. Peripheral lymphoid organs are specific sites of scrapie agent replication outside the CNS $(2,3)$. Their importance for neuroinvasion after inoculation at the periphery was suspected in light of the observation that splenectomy and other methods that reduce peripheral lymphoid structures delay the onset of clinical manifestations $(4,5)$. $\mathrm{B}$ cell-deficient mice resist usual doses of scrapie agent (6), which in fact may be related to impaired follicular dendritic cell (FDC) differentiation in those animals ( 7 , 8). Indeed, certain models of FDC deficiency (TNF- $\alpha$ gene disruption, or treatment with soluble recombinant lymphotoxin- $\beta$ receptor [LT $\beta \mathrm{R}-\mathrm{Ig}]$ ]) display resistance to scrapie (8-10), whereas constitutive expression of cellular PrP on FDCs but not on lymphoid cells correlates with scrapie susceptibility (8). Because PrP expression is required for replication of the scrapie agent (11), FDCs are thought to be an essential site of amplification outside the nervous system.

Nevertheless, the mechanisms of neuroinvasion remain obscure, and some observations suggest that involvement of another cell compartment could follow accumulation in the germinal centers of lymphoid organs. Recently, the involvement of cells other than FDCs, possibly of myeloid origin, has been put forward as a possible factor in prion neuroinvasion from the periphery (12). When scrapie-infected mice were treated with LT $\beta \mathrm{R}-\mathrm{Ig}$, the pathological conformer of PrP $\left(\mathrm{PrP}^{\mathrm{Sc}}\right)$ was shown to persist in the spleen in spite of the absence of FDCs (10). In the present study, we tested the hypothesis that $\mathrm{CD} 11 \mathrm{c}^{+}$dendritic cells, which are clearly distinct from FDCs based on their phenotype, location, and functions $(13,14)$ and are specialized in capturing and carrying antigens, might accumulate prions and transfer them to the CNS.

\section{Methods}

Scrapie inoculation. The 139A strain was chosen because first, it had been cloned, and second, its incubation period after peripheral inoculation is shorter and less variable than with other strains; finally, its titer in spleens is among the highest (15). The clonal 139A scrapie strain was kindly provided by R.H. Kimberlin. 
It has been passaged serially by intracerebral injection of C57BL/6J female mice (The Jackson Laboratory, Bar Harbor, Maine, USA). At the time of clinical disease, brains were removed under sterile conditions and $10 \%$ homogenates were prepared in PBS. All homogenates were stored at $-70^{\circ} \mathrm{C}$ before use. To obtain the spleens for cell fractionation, C57BL/ 6 female mice were injected intraperitoneally with $0.025 \mathrm{ml}$ of a $1 \%$ homogenate of 139A-positive brain. Spleens were harvested 10-13 weeks after injection. Five of the injected mice were allowed to proceed to clinical disease, and the incubation period of $175 \pm 3$ days was that expected for the $139 \mathrm{~A}$ strain in intraperitoneally injected C57BL/6 mice.

Cell subset fractionation. Spleens from 139A-infected mice were immediately injected with $3 \mathrm{ml}$ of $1 \mathrm{mg} / \mathrm{ml}$ collagenase D (Boehringer Mannheim Biochemicals Inc., Mannheim, Germany) in PBS and then left to incubate 40 minutes at $37^{\circ} \mathrm{C}$. After filtration on 100 $\mu \mathrm{m}$ mesh, mononuclear cells were obtained by centrifugation on Lympholyte-M (Cedarlane Laboratories Ltd., Hornby, Ontario, Canada) and washed with PBS containing $0.5 \% \mathrm{BSA}$ and $2.5 \mathrm{mM}$ sodium EDTA. All steps of magnetic cell sorting (MACS; Miltenyi Biotec, Auburn, California, USA) intracerebral were performed in this buffer. After blocking with $10 \%$ mouse serum for 20 minutes at room temperature, dendritic cells were enriched by positive selection with antiCD11c-conjugated magnetic particles according to the manufacturer's instructions. A B cell-enriched fraction was prepared by depleting the flow-through cells from the CD11c sorting with anti-CD90 and anti-CD43 magnetic particles. Total splenocytes as well as cell fractions were analyzed by cytofluorimetry using antiB220,-CD3, -CD11c, and -CD11b phycoerythrin- or FITC-conjugated antibodies (PharMingen International, San Diego, California, USA).

Infectivity measurements. Serial tenfold dilutions of lysates from the various fractionated spleen cell preparations were injected intracerebrally $(0.025 \mathrm{ml})$ into CD-1 mice and end-point titers determined. Titers were calculated by the Reed-Muench formula.

For confirming scrapie diagnosis, in seven recipient animals from the different groups (see below) sacrificed either at terminal scrapie or after sufficient incubation, presence of infectious agent was searched for by direct intracerebral passage of $0.025 \mathrm{ml}$ of $1 \%$ brain homogenate into CD-1 mice (six to eight mice per inoculum).

Adoptive cell transfer. Immediately after fractionation, cells were washed and suspended in PBS $(200 \mu \mathrm{l}$ per

\section{Table 1}

Titers of scrapie infectivity in spleen cell populations from C57BL/6 mice injected with the $139 \mathrm{~A}$ scrapie strain

\begin{tabular}{lcc}
\hline Cell preparation & Purity & $\begin{array}{c}\text { Titer } \\
\left(\log _{10} \mathrm{U} / 10^{6} \text { cells }\right)\end{array}$ \\
Total mononuclear cells & $46 \%$ B cells / 38\% T cells / & 3.9 \\
& $2.5 \%$ dendritic cells \\
Dendritic cells (CD11 c $\left.c^{+}\right)$ & $87 \%$ & 4.55 \\
B cells & $94 \%$ & 3.45 \\
\hline
\end{tabular}

injection), filtered on a $70-\mu \mathrm{m}$ mesh, and injected intravenously in the tail of 8 -week-old $R A G-1^{0 / 0}$ mice (The Jackson Laboratory). Animals received three consecutive injections at 1-week intervals of total mononuclear spleen cells $\left(2 \times 10^{7}\right.$ per injection), B cells $\left(10^{7}\right.$ per injection), or CD $11 \mathrm{c}^{+}$dendritic cells $\left(10^{6}\right.$ per injection). In parallel, groups of mice were also inoculated intravenously with the same amounts of B cells or total mononuclear spleen cells that had been killed by three cycles of freezing and thawing. Because limited amounts of splenic $\mathrm{CD} 11 \mathrm{c}^{+}$dendritic cells could be obtained, they were all injected as live cells.

Disease monitoring. Recipient RAG-10/0 mice were monitored weekly for clinical disease starting at 10 weeks after infection by observing their activity levels and competence on a set of parallel bars as described previously (16). The scrapie incubation period was designated as the day on which the mouse had shown clinical symptoms for the third consecutive week. At this stage, they were within 1-4 weeks of death. Diagnosis of scrapie was confirmed by detection of $\mathrm{PrP}^{\mathrm{Sc}}$ and by intracerebral passage of $0.025 \mathrm{ml}$ of $1 \%$ brain homogenate into CD-1 mice.

Detection of brain $\mathrm{PrP}^{S c}$. One half brain sample from each animal was processed for $\mathrm{PrP}^{\mathrm{Sc}}$ isolation based on detergent insolubility, using the TL100 procedure as described previously (17). Proteinase $\mathrm{K}$ was added to a concentration of $50 \mu \mathrm{g} / \mathrm{ml}$ and left to incubate for 10 minutes at room temperature. Approximately 0.075 brain equivalents of each brain sample, with or without previous proteinase $\mathrm{K}$ treatment, were deposited on $12 \%$ SDS-PAGE and analyzed by Western blot using polyclonal rabbit anti-PrP antiserum 78295 (18).

Histology. Spleens were collected from sacrificed RAG- $1{ }^{0 / 0}$ mouse recipients and cut in two parts. A small part was frozen for immunofluorescence studies, and the remaining part was fixed in paraformaldehyde.

Table 2

Fate of RAG-10/0 mice after intravenous inoculation with different 139A-positive spleen cell populations obtained from scrapie-infected mice

\begin{tabular}{lcccc}
\hline Cell preparation & No. injected cells & Total injected infectivity $\left(\log _{10} U\right)$ & Infected animals & Incubation time $($ days $)$ \\
Total mononuclear cells & $20 \times 10^{6}(\times 3)$ & 5.7 & $8 / 8$ & $209 \pm 2$ \\
Dendritic cells $\left(\mathrm{CD} 11 \mathrm{c}^{+}\right)$ & $10^{6}(\times 3)$ & 5.0 & $3 / 3^{\mathrm{A}}$ & $266 \pm 17$ \\
B cells $(\mathrm{CD} 43$ depleted) & $10 \times 10^{6}(\times 3)$ & 4.9 & $8 / 9$ & $275 \pm 13$ \\
\hline
\end{tabular}

Aln a fourth mouse injected with the CD11 $c^{+}$-enriched fraction that died accidentally at day 186 , searches for brain PrPSc were unequivocally positive, as well as scrapie infectivity (Table 3 ). 


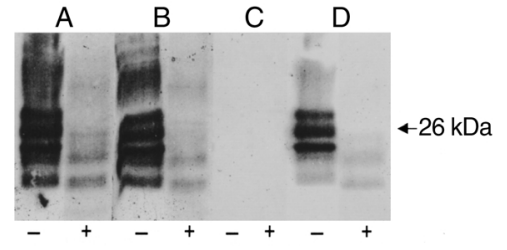

\section{Figure 1}

Western blot detection of PrPSc (PrP-res) in brain extracts from four mice inoculated with scrapie-infected spleen cell fractions. Each lane was loaded with 0.075 brain equivalents, with $(+)$ and without $(-)$ previous treatment by proteinase $\mathrm{K}$ as described in the text. Extracts were prepared from brains of RAG-10/0 that were sacrificed at terminal disease or more than 400 days after inoculation with the following 139A-infected spleen cell preparations: (lane group A) Total mononuclear cells, (lane group B) B cell-enriched cells, (lane group C) killed B cells, and (lane group D) dendritic cells $\left(C D 11 c^{+}\right)$. Note that the extraction procedure does not recover normal PrPC because of its detergent solubility, which explains the absence of signal in lane $\mathrm{C}(-)$. Extracts in lane group $\mathrm{A}$, lane group $\mathrm{B}$, and lane group $\mathrm{D}$ display high amounts of PrP that are resistant to proteinase $K$, with a shift in apparent sizes of the PrP glycoforms that is characteristic of PrPSc.

Fixed tissue sections $(5 \mu \mathrm{m})$ were stained with hematoxylin and eosin. For PrPsc detection, sections were treated by microwave heating ( $350 \mathrm{~W}$ for 30 minutes) followed by immersion in $98 \%$ formic acid (30 minutes) and were revealed using a biotinylated anti-PrP $\mathrm{mAb}$ (clone SAF83; kindly provided by J. Grassi, Commisariat à l'Energie Atomique, Fontenay-aux-Roses, France) and then horseradish peroxidase-conjugated streptavidin followed by $\mathrm{H}_{2} \mathrm{O}_{2} /$ diaminobenzidin reaction. Frozen sections $(5 \mu \mathrm{m})$ were fixed in acetone, rehydrated in PBS 0.5\% BSA, and stained sequentially with biotinylated rat anti-mouse CD11c (PharMingen), streptavidin-cychrome5 (Jackson ImmunoResearch Laboratories Inc., West Grove, Pennsylvania, USA), FITC-conjugated rat anti-CD3, and phycoerythrin-conjugated rat anti-CD19 (PharMingen). Slides were analyzed using an LSM510 confocal microscope (Carl Zeiss, Esslingen, Germany).

\section{Results}

Scrapie infectivity in spleen cell fractions. Spleens were collected from C57BL/6 mice 10-13 weeks after intraperitoneal infection by the 139A scrapie strain, i.e., at a preclinical stage, and cell fractions were isolated as described above. Three spleen cell preparations were utilized as follows: a total mononuclear splenocyte population, a fraction enriched with CD11c/MHC class II(I$\mathrm{Ab})$-positive cells corresponding to dendritic cells, and a B cell-enriched fraction. On a per cell basis, the infectivity titer found in the CD11 $\mathrm{c}^{+}$dendritic cell fraction was more than 12 -fold

\section{Table 3} passage into $C D-1$ mice that found in the B cell-enriched fraction, and four to five times the titer found in the total mononuclear cell population (Table 1). The high infectivity rate found in the $\mathrm{CD} 11 \mathrm{c}^{+}$fraction suggests that dendritic cells are possible vectors of prion propagation, a hypothesis that was tested by transfer experiments of live cells.

Scrapie transmission by live spleen cell fractions. The same three spleen cell preparations were inoculated intravenously into $R A G-1^{0 / 0}$ recipients, in the form of live cells. The number of injected cells for each fraction corresponded to that found in one normal spleen. For exposing recipient animals to infected cells during a relatively prolonged time, we decided to administer three intravenous injections at 1 -week intervals. As controls, mice were injected in similar conditions with splenocyte preparations from the same infected mouse donors, after killing cells by three cycles of freezing and thawing.

Inoculation with total mononuclear spleen cells or $\mathrm{CD}_{11 \mathrm{c}^{+}}$or B cell-enriched fractions induced scrapie in all recipients, except in one animal that had received the B cell-enriched preparation (Table 2). As expected, incubation times were shorter in animals that received total mononuclear spleen cells, given that they included both $\mathrm{CD} 11 \mathrm{c}^{+}$dendritic cells and B cells together with other cell types, as well as a higher amount of infectious agent. None of five mice injected with frozen and thawed B cell lysates containing $10^{4.9}$ infectious units of the same agent showed any signs of scrapie; three of these mice were sacrificed at 310 days, and two, at 407 days. Three mice injected with $10^{5.7}$ infectious units in total mononuclear spleen cell lysates died of intercurrent infection after 232-269 days without signs of scrapie. These results merely confirm the known resistance of $R A G-1^{0 / 0}$ mice to peripheral inoculation with prions in cell lysates (6) and show that live spleen cells may, in certain conditions, transfer the disease. $\mathrm{PrP}^{\mathrm{Sc}}$ was detected in brains of all animals showing clinical signs of scrapie, including those that had received the $\mathrm{B}$ cell- and dendritic cell-enriched fractions and the total mononuclear spleen cells (Figure 1). None of those that had been injected with killed cells displayed detergent insoluble proteinase $\mathrm{K}$-resistant $\mathrm{PrP}$ in the brain. Moreover, the diagnosis of scrapie was confirmed by passage into new mice (Table 3): All tested animals that presented with clinical scrapie also displayed high cerebral infectivity

Scrapie infectivity in brains from $R A G-10 / 0$ recipient mice: incubation times after intracerebral

\begin{tabular}{lccc}
$\begin{array}{l}\text { Donor } R A G-1^{0 / 0} \text { mice } \\
\text { originally inoculated with }\end{array}$ & $\begin{array}{c}\text { Clinical status of } \\
\text { the } R A G-1^{0 / 0} \text { mice }\end{array}$ & $\begin{array}{c}\text { Number of scrapie-positive } \\
\text { CD-1 mouse recipients }\end{array}$ & $\begin{array}{c}\text { Incubation periods } \\
\text { (days) }\end{array}$ \\
Total spleen cells & Scrapie & $7 / 7$ & $131 \pm 3$ \\
Total spleen cells & Scrapie & $6 / 6$ & $134 \pm 2$ \\
B cells & Scrapie & $7 / 7$ & $133 \pm 3$ \\
B cells & Scrapie & $6 / 6$ & $128 \pm 3$ \\
Dendritic cells (CD11c & Scrapie & $7 / 7$ & $134 \pm 2$ \\
Dendritic cells (CD11c & Healthy at 186 days & $8 / 8$ & $146 \pm 1$ \\
Killed B cells & Healthy at 310 days & $0 / 8$ & $>200$ \\
\hline
\end{tabular}




\section{Figure 2}

Absence of lymphoid reconstitution in mice injected with infected CD11 $c^{+}$cells. (a-d) Hematoxylin and eosin staining of paraformaldehyde-fixed sections at the same magnification. $\times 25$. A normal lymphoid architecture of the spleen is found in $R A G 1 \% 0$ mice that had been injected with live total spleen cells (a) and the $B$ cell-enriched fraction (b), but not in RAG 1\%/0 mice injected with killed spleen cells (c) or the live $\mathrm{CD} 11 \mathrm{c}^{+}$cell-enriched fraction (d). (e and $\mathbf{f}$ ) Higher magnification $(\times 1,000)$ of lymphoid follicles in spleens from mice injected with total spleen cells (e) reveals typical aspect including immunoblasts and centroblast, which is clearly different from that of nodules found in spleens from $C D 11 C^{+}$ cell-injected mice (f); the latter are made of smaller cells, essentially with a nonlymphoid morphology and likely belonging to other hematopoietic lineages. ( $\mathbf{g}$ and $\mathbf{h}$ ) Frozen sections from spleens of RAG1\%/0 mouse recipients injected with total spleen cells $(\mathrm{g} ; \times 400)$ or the CD11 $\mathrm{c}^{+}$ cell-enriched fraction $(\mathbf{h} ; \times 400)$ were triple stained with anti-CD3-FITC (green), anti-CD19-phycoerythrin (red), and anti-CD11c-Cychrome5 (blue). The absence of lymphoid structure in RAG1\% mice injected with CD11 $c^{+}$cells is confirmed on $\mathbf{h}$, which is representative of the entire spleen and shows no staining for B (CD19) and T (CD3) cells. (i and $\mathbf{j}$ ) Immunohistochemical evidence of $\mathrm{PrPSc}$ (see Methods) accumulation in spleens from $R A G 1^{\circ / 0}$ mice infected with total spleen cells $(\mathbf{i} ; \times 200)$ but not in those from mice infected with the CD11 $\mathrm{c}^{+}$ cell-enriched fraction $(\mathbf{j} ; \times 200)$.
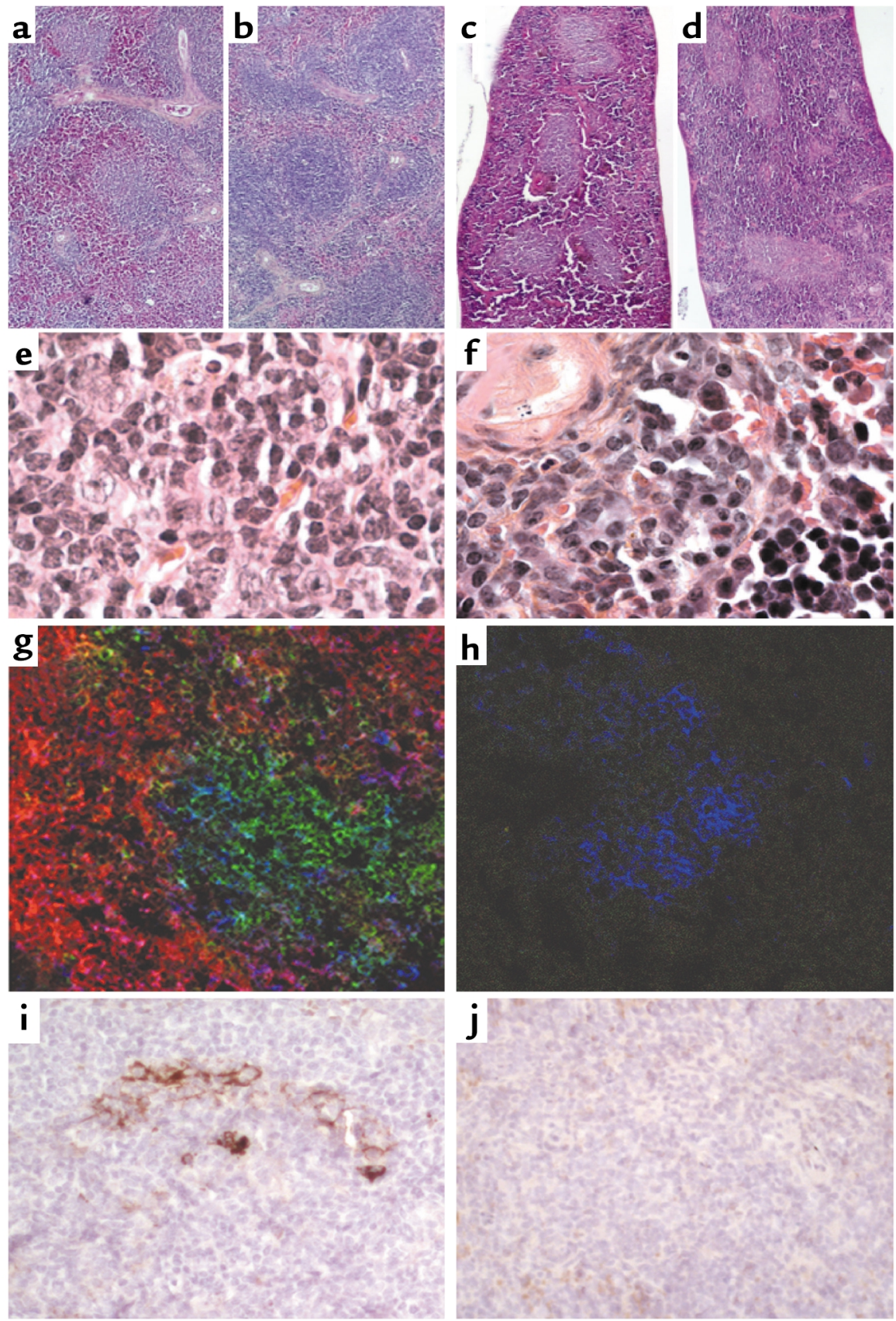

titers of scrapie agent. Interestingly, in one $R A G-1^{0 / 0}$ mouse that had received infected dendritic cells and died of intercurrent infection before the clinical onset of scrapie, a lower but still strong level of brain infectivity was detected (incubation period after passage to CD-1 mice: $146 \pm 1 ; n=8)$. No infectivity was detected in the tested mouse that had been injected with killed infected total mononuclear spleen cells as of more than 200 days after infection $(n=8)$.

Immunological status of $R A G-1^{10 / 0}$ recipients. Importantly, no evidence of lymphoid reconstitution was detected in spleens from mice injected with $\mathrm{CD} 11 \mathrm{c}^{+}$cells by histological and immunofluorescence criteria (Figure 2). Histological morphology was unequivocally identical to that of $R A G-1^{0 / 0}$ mice that had received killed cells, with a predominance of red pulp and some nonlymphoid nodules. On the other hand, mice injected with total mononuclear spleen cells or the B cell-enriched fraction had numerous lymphoid follicles in the spleen that stained for $\mathrm{B}$ and $\mathrm{T}$ cell markers, showing that B cell enrichment was only partial and that precursor cells of both lymphoid lineages were contained in the inoculum. As shown by immunochemical study after treatment in order to denature $\operatorname{PrPC}^{\mathrm{C}}$ and enhance $\mathrm{Pr} P \mathrm{Pc}$ immunoreactivity (Figure 2, $i$ and $j$ ), prion protein was detected in the spleens of mice with lymphoid reconstitution but not in those that received the $\mathrm{CD} 11 \mathrm{c}^{+}$ dendritic cell fraction. This suggests that a replication 
step in the spleen was involved in the former case but not after injection of infected dendritic cells alone.

\section{Discussion}

According to published data, mature B cells and, secondarily, germinal centers and FDC networks, are required for the development of clinical scrapie after peripheral injection using usual pathogenic doses (6). Recently published results suggest that splenic B lymphocytes are not able to replicate prions by themselves (19); infectivity detected in B cells from infected animals is likely acquired passively through their close contacts with FDCs. A possible specific role of B cells may not be evaluated in the present study because the corresponding cell preparation included enough lymphoid precursors to allow complete reconstitution of $\mathrm{B}$ and $\mathrm{T}$ cell structures. Thus, as also observed in RAG-10/0 mice inoculated with infected total mononuclear spleen cells, reconstitution of lymphoid architecture allows accumulation of scrapie agent in the spleen and subsequent propagation to the CNS, which may not occur in nonreconstituted animals. On the other hand, by injecting a fraction strongly enriched with live infected CD $11 \mathrm{c}^{+}$splenic cells, the infectious process bypassed the accumulation step in germinal centers. In the latter model, no PrPSc accumulation was observed on spleen sections at terminal disease, in spite of the presence of CD11 $\mathrm{c}^{+}$cells (Figure $2, h$ and $j$ ), suggesting that dendritic cells may be sufficient for carrying prions to sites of neuroinvasion but would not replace FDCs for replication and accumulation in lymphoid organs. Although we could not inject mice with a lysate of purified $\mathrm{CD} 11 \mathrm{c}^{+}$dendritic cells, owing to the limited amount of such cells that can be recovered from spleens, inoculation by the same route using killed B cells containing similar levels of infectivity did not induce clinical disease. This control result only confirms the well-demonstrated resistance of $R A G-1^{0 / 0}$ mice to peripheral inoculation with scrapie agent (6).

Given that $\mathrm{CD} 11 \mathrm{c}^{+}$cells are present in spleens of nonreconstituted $R A G-1^{0 / 0}$ mice, there should be a certain requirement(s) for these cells to capture sufficient amounts of scrapie agent and allow neuroinvasion that are not fulfilled in the absence of lymphoid follicles. Such requirements might be the presence of mature FDCs, where prions could replicate before being delivered to dendritic cells. However, in certain conditions the scrapie agent seems able to reach the CNS without or with little help from FDCs: after intraperitoneal inoculation by usual doses, infectivity and proteaseresistant prion protein and spongiform changes were found in the brains of certain B cell/FDC-deficient mice in spite of the absence of clinical symptoms $(6$, 20); moreover, much higher doses injected in similar conditions provoked clinical disease in B cell/FDCdeficient mice (7). Finally, certain models of FDC-deficient mice are susceptible to scrapie infection after peripheral inoculation $(6,8,21)$. The hypothesis of a participation of $\mathrm{CD} 11 \mathrm{c}^{+}$dendritic cells in the process of neuroinvasion is compatible with what was observed when FDC maturation was suppressed by administration of LT $\beta R$-Ig: In these models, as stressed by Montrasio et al. (9) and Mabbott et al. (10), impaired replication of scrapie agent at the periphery could be related not only to FDC but also to dendritic cell defect.

A role for $\mathrm{CD} 11 \mathrm{c}^{+}$dendritic cells in the pathogenesis of transmissible spongiform encephalopathies in normal animals is supported by our finding of a comparatively high level of infectivity in this cell fraction. Fragments of FDC dendrites may have contaminated cell fractions and contributed to their infectivity; however, this would mainly concern $B$ cells because of their close contacts with FDC networks; CD11 $\mathrm{c}^{+}$dendritic cells, which predominate in $\mathrm{T}$ cell areas, were found to bear 12 times more infectious agent per cell. In accordance with this result, non-B/non-T stromal spleen cells from scrapie-infected mice were found to carry infectivity (22, 23). Moreover, in scrapie-infected mice treated with LT $\beta$ R-Ig, the pathological conformer of the prion protein $\left(\mathrm{PrP}^{\mathrm{Sc}}\right)$ was shown to persist in the spleen in spite of the disappearance of mature FDCs (10). PrPsc aggregates might accumulate on the surface of FDC dendrites, from where they would be released to other cells permitting dissemination $(12,24)$. Although other cells, such as tingible body macrophages (24), are potential vectors of prion propagation from the lymphoid follicles, CD $11 \mathrm{c}^{+}$dendritic cells are sufficient for neuroinvasion in $R A G-1^{10 / 0}$ recipients.

Although our adoptive transfer model in RAG-10/0 mice shows that dendritic cells are able to carry the scrapie agent from the vascular compartment to the CNS, the precise role of these cells in natural infection remains to be clarified.

The respective participation of FDCs and $\mathrm{CD} 11 \mathrm{c}^{+}$ dendritic cells might vary according to the conditions of infection and the route of neuroinvasion. It might also depend in part on the scrapie strains used, which would explain discrepancies observed in previous studies. The present results need to be confirmed with other prion strains. Using the 139A strain, we found that CD11 $\mathrm{c}^{+}$dendritic cells collected from mice during the incubation period are able to carry the infection progressively to the CNS, without detectable accumulation in the spleen. Whether these cells replicate the scrapie agent, modify its nature, or acquire it passively, and how they interact directly or indirectly with nervous cells, remain to be established. A more complete understanding of the steps and the cells involved in prion spreading from the periphery to the brain may provide a pharmacological gatekeeper that could be used to stop the movement of infectivity.

\section{Acknowledgments}

This study was supported by grants from the NIH (AG15408 and AR02594) and by the French ESST99 research program. We are most grateful to Marie KoscoVilbois for helpful discussions and advice; to Claude 
Carnaud, Olivier Lantz, and Patricia Lemarchand for critical reading of the manuscript; and to Marjorie Thouroude and Olivier Babin for technical assistance.

1. Prusiner, S.B. 1997. The prion diseases of humans and animals. In The molecular and genetic basis of neurological diseases. R.N. Rosenberg, S.B. Prusiner, S. Di Mauro, and R.L. Barchi, editors. Butterworth-Heinemann. Boston, Massachusetts, USA. 165-186.

2. Eklund, C.M., Kennedy, R.C., and Hadlow, W.J. 1967. Pathogenesis of scrapie virus infection in the mouse. J. Infect. Dis. 117:15-22.

3. Kimberlin, R.H., and Walker, C.A. 1979. Pathogenesis of mouse scrapie: dynamics of agent replication in spleen, spinal cord and brain after injection by different routes. J. Comp. Pathol. 89:551-562.

4. Fraser, H., and Dickinson, A.G. 1978. Studies of the lymphoreticular system in the pathogenesis of scrapie: the role of spleen and thymus. J. Comp. Pathol. 88:563-573.

5. Outram, G.W., Dickinson, A.G., and Fraser, H. 1974. Reduced susceptibility to scrapie in mice after steroid administration. Nature. 249:855-856.

6. Klein, M.A., et al. 1997. A crucial role for B cells in neuroinvasive scrapie. Nature. 390:687-690.

7. Klein, M.A., et al. 1998. PrP expression in B lymphocytes is not required for prion neuroinvasion. Nat. Med. 4:1429-1433.

8. Brown, K.L., et al. 1999. Scrapie replication in lymphoid tissues depends on prion protein-expressing follicular dendritic cells. Nat. Med. 5:1308-1312.

9. Montrasio, F., et al. 2000. Impaired prion replication in spleens of mice lacking functional follicular dendritic cells. Science. 288:1257-1259.

10. Mabbott, N.A., Mackay, F., Minns, F., and Bruce, M.E. 2000. Temporary inactivation of follicular dendritic cells delays neuroinvasion of scrapie. Nat. Med. 6:719-720.

11. Blättler, T., et al. 1997. PrP-expressing tissue required for transfer of scrapie infectivity from spleen to brain. Nature. 389:69-73.
12. Manuelidis, L., et al. 2000. Follicular dendritic cells and dissemination of Creutzfeldt-Jakob disease. J. Virol. 74:8614-8622.

13. Banchereau, J., et al. 2000. Immunobiology of dendritic cells. Annu. Rev. Immunol. 18:767-811.

14. Kosco-Vilbois, M.H., Bonnefoy, J.Y., and Chvatchko, Y. 1997. The physiology of murine germinal center reactions. Immunol. Rev. 156:127-136.

15. Carp, R.I., Meeker, H., and Sersen, E. 1997. Scrapie strains retain their distinctive characteristics following passages of homogenates from different brain regions and spleen. J. Gen. Virol. 78:283-290.

16. Carp, R.I., Callahan, S.M., Sersen, E.A., and Moretz, R.C. 1984. Preclinical changes in weight of scrapie-infected mice as a function of scrapie agent-mouse strain combination. Intervirology. 21:61-69.

17. Kascsak, R.J., Fersko, R., Pulgiano, D., Rubenstein, R., and Carp, R.I. 1997. Immunodiagnosis of prion disease. Immunol. Invest. 26:259-268.

18. Kascsak, R.J., et al. 1987. Mouse polyclonal and monoclonal antibody to scrapie-associated fibril proteins. J. Virol. 61:3688-3693.

19. Montrasio, F., et al. 2001. B lymphocyte-restricted expression of prion protein does not enable prion replication in prion protein knockout mice. Proc. Natl. Acad. Sci. USA. 98:4034-4037.

20. Frigg, R., Klein, M.A., Hegyi, I., Zinkernagel R.M., and Aguzzi, A. 1999. Scrapie pathogenesis in subclinically infected B-cell-deficient mice. $J$. Virol. 73:9584-9588.

21. Mabbott, N.A., et al. 2000. Tumor necrosis factor alpha-deficient, but not interleukin-6-deficient, mice resist peripheral infection with scrapie. J. Virol. 74:3338-3344.

22. Clarke, M.C., and Kimberlin, R.H. 1984. Pathogenesis of mouse scrapie: distribution of agent in the pulp and stroma of infected spleens. Vet. Microbiol. 9:215-225.

23. Raeber, A.J., et al. 1999. PrP-dependent association of prions with splenic but not circulating lymphocytes of scrapie-infected mice. EMBO J. 18:2702-2706

24. Jeffrey, M., McGovern, G., Goodsir, C.M., Brown, K.L., and Bruce, M.E. 2000. Sites of prion protein accumulation in scrapie-infected mouse spleen revealed by immune-electron microscopy. J. Pathol. 191:323-332. 